\title{
Herramienta Computacional para la Enseñanza de la Evaluación del Campo Eléctrico en Instalaciones Industriales
}

\author{
Andrea P. Rossi ${ }^{1}$ y Patricia N. Baldini ${ }^{2}$ \\ Universidad Tecnológica Nacional, Facultad Regional Bahía Blanca, (1) Departamento de Ingeniería \\ Eléctrica, (2) Departamento de Ingeniería Electrónica, 11 de Abril 461, B8000LMI Bahía Blanca, \\ Buenos Aires-Argentina (e-mail: aprossi@frbb.utn.edu.ar, pnbaldi@frbb.utn.edu.ar)
}

\begin{abstract}
Resumen
Se presenta en este trabajo una rutina desarrollada en Matlab ${ }^{\mathrm{TM}}$ para la enseñanza de la evaluación del campo eléctrico generado por líneas eléctricas existentes en instalaciones industriales. Considerando el plano perpendicular a la traza, la rutina calcula el valor del campo eléctrico de baja frecuencia en cualquier punto del espacio circundante a una línea eléctrica de cualquier configuración geométrica y permite visualizar el comportamiento temporal en estado estacionario del campo eléctrico. Utilizando esta rutina, se analizó en aula el comportamiento del campo eléctrico generado por una línea trifásica para diferentes configuraciones de sus conductores. Los resultados gráficos son concordantes con los provistos en trabajos publicados por otros autores. La aplicación del programa en la enseñanza facilitó la comprensión por parte de los alumnos del comportamiento del campo eléctrico en cercanías de líneas eléctricas.
\end{abstract}

Palabras claves: herramienta computacional, enseñanza, campo eléctrico, líneas eléctricas, instalaciones industriales

\section{Computational Tool to Teach the Behaviour of the Electric Field in Industrial Installations}

\begin{abstract}
In this work, a program developed in Matlab ${ }^{\mathrm{TM}}$ to teach how to evaluate the electric field of electric lines in industrial installations is presented. Considering a plane perpendicular to the line route, the program calculates low frequency electric field values for any spatial point around an electric line of any geometrical configuration, and allows observing the temporal behaviour of the electric field. The behaviour of the electric field for a three phase electric line with different geometrical configurations was simulated in class. The program graphic results were similar to those provided by other authors. The use of the program in class facilitated the comprehension by the students of the behaviour of electric fields near power lines.
\end{abstract}

Keywords: computational tool, teach, electric field, electric lines, industrial installations 


\section{INTRODUCCIÓN}

Todo proceso industrial de gran tamaño conlleva la utilización de energía eléctrica para su desarrollo. En estos casos, la presencia de instalaciones eléctricas con líneas de frecuencias de 50 [hz] e importantes valores de tensión, genera en una industria campos electromagnéticos variables de muy bajas frecuencias que pueden afectar el medioambiente, y tener un efecto negativo sobre quienes trabajan en ellas (IRPA, 1994; Dawson et al., 2000a; Dawson et al., 2000b; Arnera y Vernieri, 2001; Vernieri et al., 2001; Vernieri et al., 2002), lo cual ha ha conducido a la realización y normalización de métodos de medición (Parraud et al., 1992; IEEE Std. 644, 1994; Resolución ENRE 1724/98, 1998; Havas, 2002; Arnera et al., 2003) y ha intensificado la realización de estudios multidisciplinares en universidades y centros de investigación sobre la naturaleza de los mismos y sus posibles efectos (Cvetkovic et al., 2006; Battisti et al., 2007), propiciando el desarrollo de modelos de simulación (Deno, 1976; Tiebin Zhao y Comber, 2000; Abdel-Salam y Abdel-Aziz, 2001; Manco et al., 2001; Saadetdin y Salih Mami, 2003), habiéndose hecho necesaria, además, la transferencia de los conocimientos adquiridos a la enseñanza de grado universitaria, dada su importancia actual. Dentro de este contexto, cobran protagonismo herramientas computacionales de cálculo y graficación (Mirotznik y Prather, 1997; Correia de Barros, 1997; Que y Sebo, 2000) que ayuden a la transmisión de conceptos difíciles de interpretar, facilitando la comprensión del fenómeno bajo estudio por parte de los alumnos.

En este sentido, y a partir de un trabajo anterior (Rossi y Montero, 2005), en el que se analiza la validez de los resultados del modelo de cálculo propuesto por Angeloni (2000), se desarrolló un programa que implementa dicho método utilizando las herramientas de cálculo y graficación de Matlab ${ }^{\mathrm{TM}}$, teniendo como objetivos los siguientes, fuertemente orientados hacia el alumno: a) facilitar el aprendizaje del comportamiento del campo eléctrico en el caso particular de líneas eléctricas, y a su vez b) mostrar la utilidad e importancia de la computación aplicada para el análisis de los resultados de un modelo en estudios de ingeniería.

\section{REPRESENTACIÓN MATEMÁTICA DEL CAMPO ELÉCTRICO DE LÍNEAS DE ENERGÍA}

Bajo determinadas suposiciones es posible representar el campo eléctrico de una línea eléctrica en un punto genérico $P$ de coordenadas $(x, y)$ sobre el plano $(X, Y)$ transversal a la línea (Fig. 1), mediante: 1) dos componentes espaciales $e_{x}$ (horizontal) y $e_{y}$ (vertical); 2) el lugar geométrico de la elipse obtenida a partir de las componentes espaciales (Deno, 1976). Conocida la distribución espacial de una línea compuesta por $\mathrm{n}$ haces de conductores, cada uno de ellos de ubicación $\left(\mathrm{x}_{\mathrm{k}}, \mathrm{y}_{\mathrm{k}}\right)$ en $[\mathrm{m}]$, con radio equivalente $r_{j}$ en $[m]$ y tensión $v_{j}$ en $[k V r m s]$, las componentes espaciales en [kVrms/m] del campo eléctrico se expresan (Angeloni, 2000; Rossi y Montero, 2005):

$$
\begin{aligned}
& e_{x}(x, y)=\sum_{k=1}^{n} \frac{4 y_{k}\left(x-x_{k}\right)}{\left(x-x_{k}\right)^{2}\left[\left(x-x_{k}\right)^{2}+2\left(y^{2}+y_{k}^{2}\right)^{2}\right]+\left(y^{2}-y_{k}^{2}\right)^{2}} \sum_{j=1}^{n} P_{k j}^{-1} v_{j} \\
& e_{y}(x, y)=\sum_{k=1}^{n} \frac{-2 y_{k}\left[\left(x-x_{k}\right)^{2}-y^{2}+y_{k}^{2}\right]}{\left.\left(x-x_{k}\right)^{2}+2\left(y^{2}+y_{k}^{2}\right)^{2}\right]+\left(y^{2}-y_{k}^{2}\right)^{2}} \sum_{j=1}^{n} P_{k j}^{-1} v_{j}
\end{aligned}
$$

La ubicación $\left(\mathrm{x}_{\mathrm{k}}, \mathrm{y}_{\mathrm{k}}\right)$ en [m] de cada haz de conductores en el plano $(\mathrm{X}, \mathrm{Y})$ transversal a la línea, se determina considerando al eje $X$ coincidente con el plano de tierra, y al eje $Y$ coincidente con el eje geométrico vertical de la disposición espacial de los haces que conforman la línea eléctrica.

Los coeficientes adimensionales $\mathrm{P}_{\mathrm{kj}}^{-1}$, denominados "coeficientes de Maxwell” (Angeloni, 2000), se obtienen de aplicar la "Teoría de la Imagen", que considera para cada conductor ubicado a una distancia "L" en [m] por encima del suelo, un conductor imagen con carga de signo opuesto ubicado 
a una distancia igual por debajo del mismo. Los coeficientes $P_{k j}^{-1}$ dependen de $\bar{H}_{i j}$ y $\bar{D}_{i j}$, que son la distancia media geométrica en [m] entre el conductor "i" y el conductor imagen "-j", y la distancia media geométrica en [m] entre el conductor "i" y el "j", respectivamente (Fig. 1).

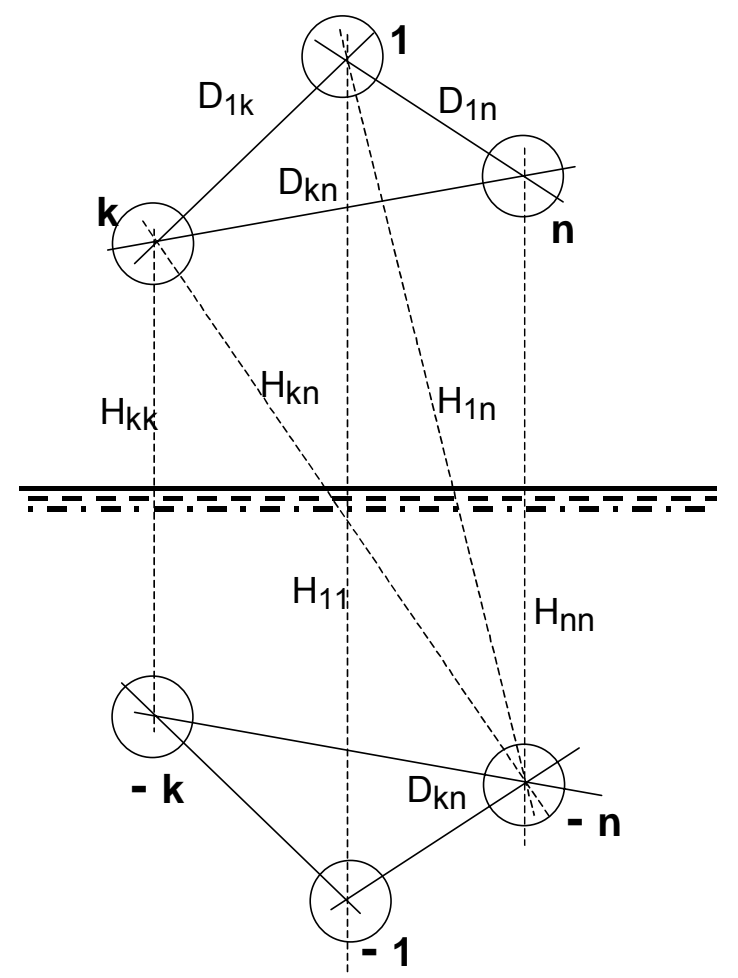

Fig. 1: Distancias medias geométricas.

La ecuación matricial (3) muestra cómo calcular los coeficientes $\mathrm{P}_{\mathrm{kj}}^{-1}$ (Angeloni, 2000).

\begin{tabular}{|l|l|l|l|l|}
\hline $\mathrm{P}_{11}^{-1}$ & $\cdots$ & $\mathrm{P}_{1 \mathrm{k}}^{-1}$ & $\cdots$ & $\mathrm{P}_{1 \mathrm{n}}^{-1}$ \\
\hline$\cdots$ & $\cdots$ & $\cdots$ & $\cdots$ & $\cdots$ \\
\hline $\mathrm{P}_{\mathrm{k} 1}^{-1}$ & $\cdots$ & $\mathrm{P}_{\mathrm{kk}}^{-1}$ & $\cdots$ & $\mathrm{P}_{\mathrm{kn}}^{-1}$ \\
\hline$\cdots$ & $\cdots$ & $\cdots$ & $\cdots$ & $\cdots$ \\
\hline $\mathrm{P}_{\mathrm{n} 1}^{-1}$ & $\cdots$ & $\mathrm{P}_{\mathrm{nk}}^{-1}$ & $\cdots$ & $\mathrm{P}_{\mathrm{nn}}^{-1}$ \\
\hline
\end{tabular}

\begin{tabular}{|c|c|c|c|c|}
\hline $\ln \left(\frac{\mathrm{H}_{11}}{\mathrm{r}_{1}}\right)$ & $\ldots$ & $\ln \left(\frac{\mathrm{H}_{1 \mathrm{k}}}{\mathrm{D}_{1 \mathrm{k}}}\right)$ & $\ldots$ & $\ln \left(\frac{\mathrm{H}_{1 \mathrm{n}}}{\mathrm{D}_{1 \mathrm{n}}}\right)$ \\
\hline$\ldots$ & $\cdots$ & $\ldots$ & $\cdots$ & $\ldots$ \\
\hline $\ln \left(\frac{\mathrm{H}_{\mathrm{k} 1}}{\mathrm{D}_{\mathrm{k} 1}}\right)$ & $\cdots$ & $\ln \left(\frac{\mathrm{H}_{\mathrm{kk}}}{\mathrm{r}_{\mathrm{k}}}\right)$ & $\cdots$ & $\ln \left(\frac{\mathrm{H}_{\mathrm{kn}}}{\mathrm{D}_{\mathrm{kn}}}\right)$ \\
\hline$\ldots$ & $\cdots$ & $\cdots$ & $\cdots$ & $\cdots$ \\
\hline $\ln \left(\frac{\mathrm{H}_{\mathrm{n} 1}}{\mathrm{D}_{\mathrm{n} 1}}\right)$ & $\cdots$ & $\ln \left(\frac{\mathrm{H}_{\mathrm{nk}}}{\mathrm{D}_{\mathrm{nk}}}\right)$ & $\cdots$ & $\ln \left(\frac{\mathrm{H}_{\mathrm{nn}}}{\mathrm{r}_{\mathrm{n}}}\right)$ \\
\hline
\end{tabular}

La tensión $v_{\mathrm{j}}$ se expresa:

$v_{j}=V_{j m a x} \cos \left(\omega t+\varphi_{j}\right)$

donde $\omega$ es la frecuencia de la red eléctrica en hertz [hz], t es la variable tiempo en segundos [s], y $\varphi$ es desfasaje del potencial del haz en radianes [rad], con respecto a un ángulo de referencia. Las ecuaciones (1) y (2) pueden expresarse: 


$$
\begin{aligned}
& e_{x}(x, y)=A_{1} V_{1 \max } \cos \left(\omega t+\varphi_{1}\right)+A_{2} V_{2 \max } \cos \left(\omega t+\varphi_{2}\right)+\ldots \ldots .+A_{n} V_{n \max } \cos \left(\omega t+\varphi_{n}\right) \\
& e_{y}(x, y)=B_{1} V_{1 \max } \cos \left(\omega t+\varphi_{1}\right)+B_{2} V_{2 \max } \cos \left(\omega t+\varphi_{2}\right)+\ldots \ldots+B_{n} V_{n \max } \cos \left(\omega t+\varphi_{n}\right)
\end{aligned}
$$

Resultando:

$$
e_{x}(x, y)=E_{x_{\max }} \cos \left(\omega t+\varphi_{x}\right) \quad e_{y}(x, y)=E_{y_{\max }} \cos \left(\omega t+\varphi_{y}\right)
$$

Donde:

$$
E_{x_{\max }}=\sqrt{\left[\sum_{i=1}^{n} A_{i} V_{i} \cos \left(\varphi_{i}\right)\right]^{2}+\left[\sum_{i=1}^{n} A_{i} V_{i} \operatorname{sen}\left(\varphi_{i}\right)\right]^{2}} \quad \varphi_{x}=\operatorname{tg}^{-1}\left[\frac{\sum_{i=1}^{n} A_{i} V_{i} \operatorname{sen}\left(\varphi_{i}\right)}{\sum_{i=1}^{n} A_{i} V_{i} \cos \left(\varphi_{i}\right)}\right]
$$

Idénticas expresiones valen para $E_{y m a x}$ y $\varphi_{y}$, respectivamente, reemplazando $A_{i}$ por $B_{j}$. El campo eléctrico total resulta, entonces, un vector que gira en el plano transversal a la línea con la frecuencia de la red, cuyo módulo describe en el tiempo una elipse en el plano $(X, Y)$, y cuya forma depende del ángulo de desfasaje entre las tensiones, y de las coordenadas del punto de cálculo (Deno, 1976; Angeloni, 2000; Rossi y Montero, 2005)

\section{IMPLEMENTACIÓN DEL MÉTODO DE CÁLCULO EN Matlab⿳亠丷厂}

Para la implementación, se procedió a discretizar los términos individuales de las ecuaciones (1) y (2) en términos adimensionales de $\omega t$, dándole valores a t con incrementos de 0,5 [ms] hasta alcanzar un ciclo (20 [ms] para sistemas de 50 [hz]). La rutina almacena los datos del problema (tensión de la línea, cantidad y ubicación de conductores y sus radios efectivos) en un archivo denominado lineas. mat mediante el comando save de Matlab $^{\mathrm{TM}}$, el que es abierto al inicio del programa (comando load ). Estos datos se almacenan según el siguiente formato:

Matriz $V(2, n): V(1, n)$ amplitud, $V(2, n)$ fase del conductor $n$-ésimo.

Matriz $\operatorname{Pos} C(2, n): \operatorname{Pos} C(1, n)$ coordenada $x n, \operatorname{Pos} C(2, n)$ coordenada yn del conductor n-ésimo.

Vector $r(n)$ : radio efectivo de cada conductor.

$\mathrm{Pxy}=[\mathrm{xk}, \mathrm{yk}]$ : vector de posición del punto donde se desea evaluar el campo.

La rutina permite visualizar: 1) el lugar geométrico descripto por el extremo del vector campo eléctrico en el tiempo, 2) la variación en el tiempo de las componentes y del campo resultante para un punto en particular, y 3) la variación espacial de las componentes y del campo resultante en función de la distancia a la línea. La rutina de Matlab ${ }^{\mathrm{TM}}$ desarrollada para la implementación del algoritmo es:

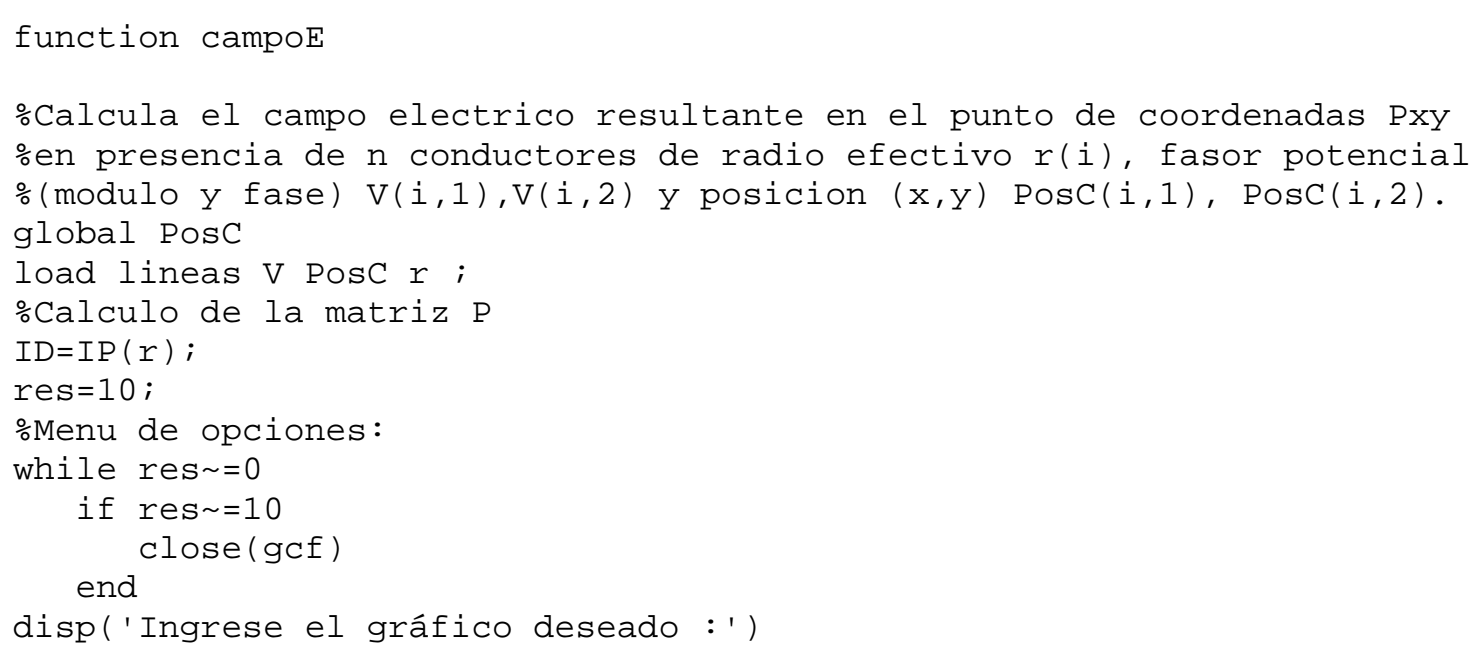




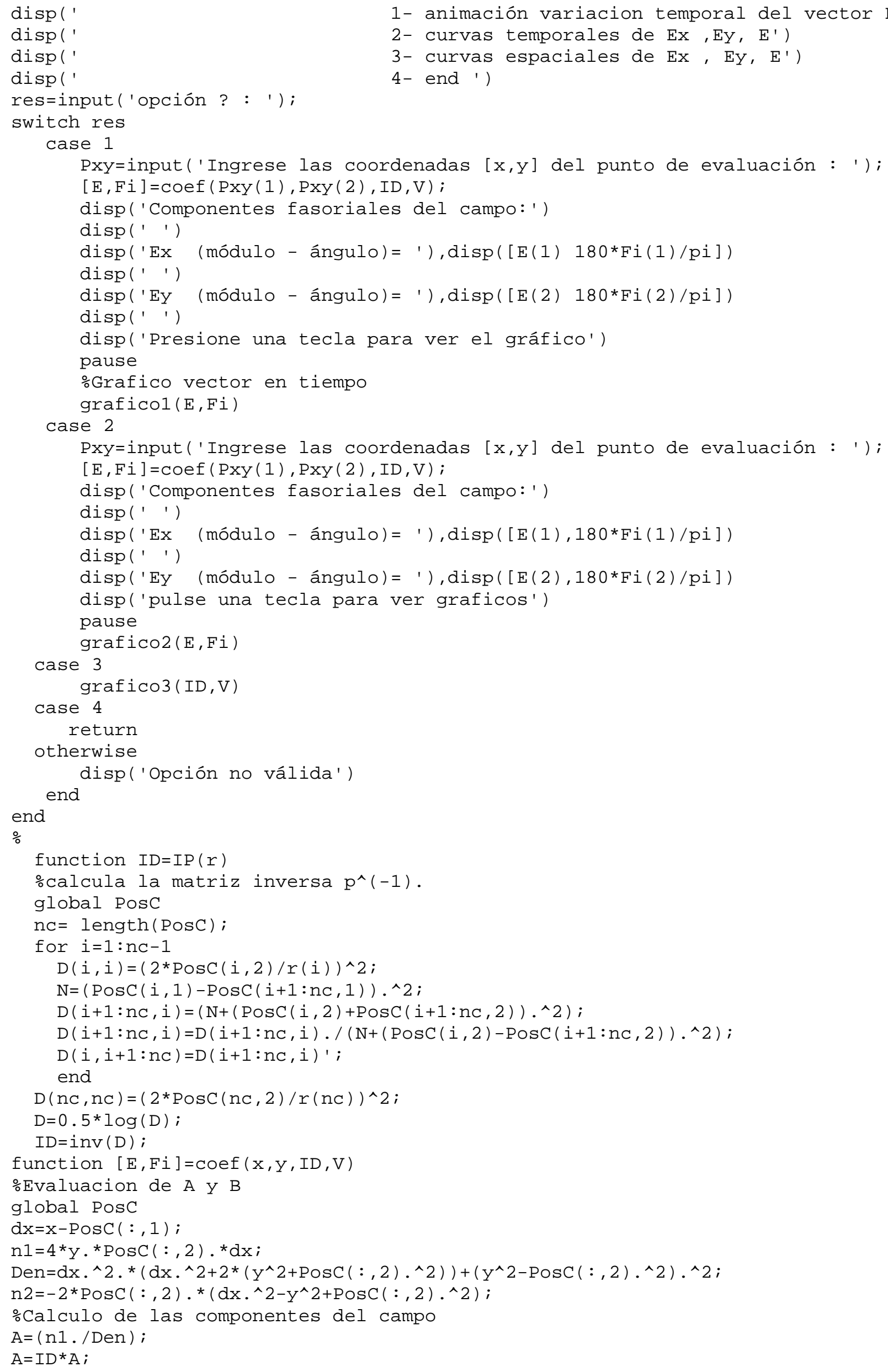




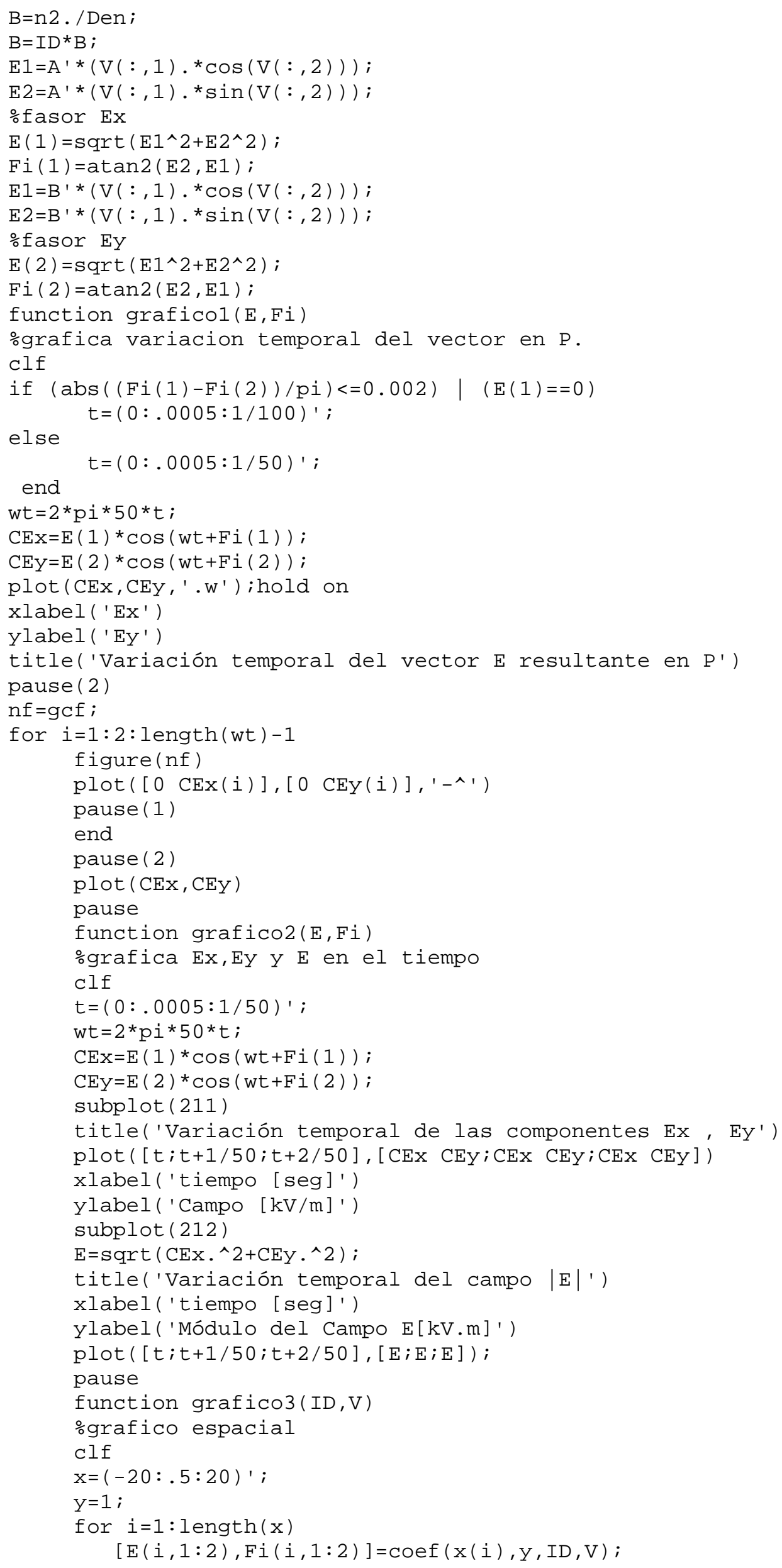




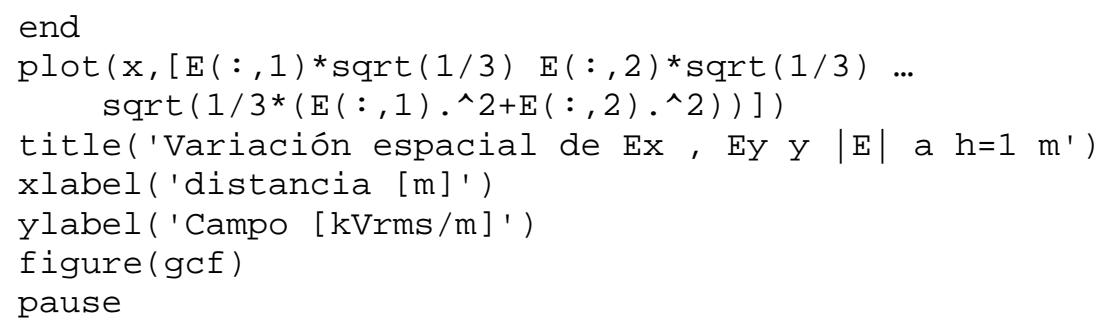

\section{RESULTADOS y DISCUSIÓN}

A modo de ejemplo, en las Figuras 2, 3 y 4 se muestran las gráficas de Matlab ${ }^{\mathrm{TM}}$ obtenidas en aula para un caso de línea trifásica de 132 [kVrms], compuesta de tres conductores de 20 [mm] de radio efectivo dispuestos en forma vertical a 9 [m], 12 [m] y 15 [m] de altura. Para una altura de 1 [m] respecto del nivel de tierra (coordenada yk) determinada por el ENRE (Ente Nacional Regulador de la Electricidad) en su Resolución ENRE 1724/98 (1998) para la medición de campos eléctricos de líneas de energía, se graficó el lugar geométrico descripto por el extremo del vector campo eléctrico en el tiempo (Fig. 2) a diferentes distancias horizontales (xk: $-6[\mathrm{~m}] \leq x \mathrm{k} \leq 4[\mathrm{~m}]$ ), fijando el origen de coordenadas sobre el nivel de tierra y coincidente con el eje vertical de los conductores.
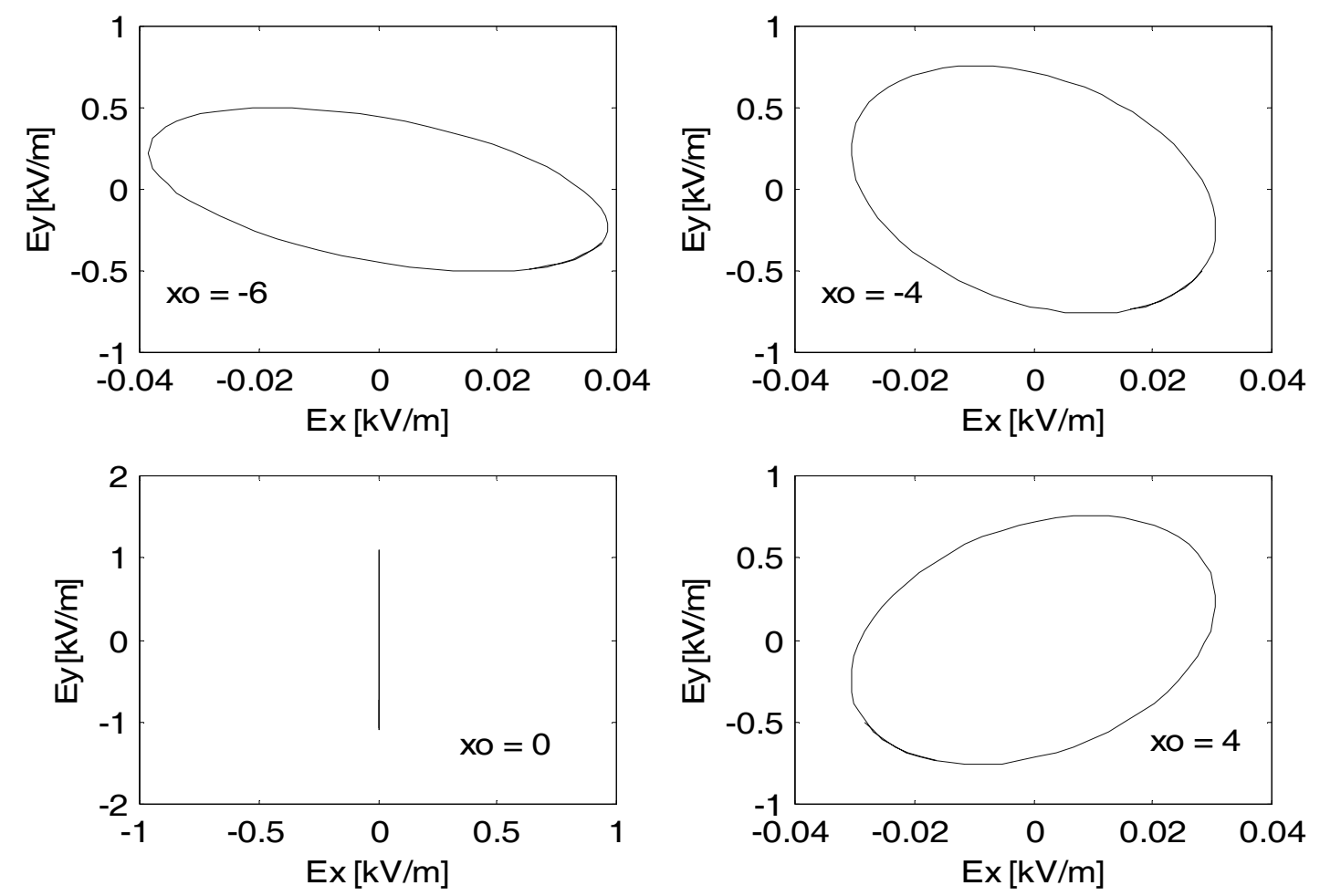

Fig. 2: Lugar geométrico de la elipse.

Los gráficos de los valores instantáneos las componentes transversales, $e_{x}$ y $e_{y}$, y del campo resultante, e, correspondientes al mismo ejemplo para un punto en el espacio de coordenadas (xk,yk) $=(5,1)[\mathrm{m}]$ se muestran para un rango temporal de 3 ciclos (Fig. 3). La Figura 4 muestra la variación espacial del vector campo eléctrico en la dirección del eje $x$ (horizontal) a una altura de 1[m] (Fig. 4).

Se repitió el análisis para otras configuraciones, variando la cantidad, características y tensión de conductores, cuyos resultados no se muestran aquí.

Se comprobó mediante simulación que, en efecto, el lugar geométrico descrito en el tiempo por el extremo del vector campo eléctrico generado por una línea eléctrica compuesta por $n$ conductores paralelos, con tensiones sinusoidales (estado estacionario), en planos transversales a los mismos, es una elipse, y que en ciertos casos esta elipse puede degenerar en un segmento o en una 
circunferencia (Deno, 1976). Mediante la utilización de la rutina, es posible observar que este resultado se mantiene cualesquiera sean las características, disposición y tensiones de los conductores paralelos, que sólo afectan la forma y orientación espacial (rotación) de la elipse.
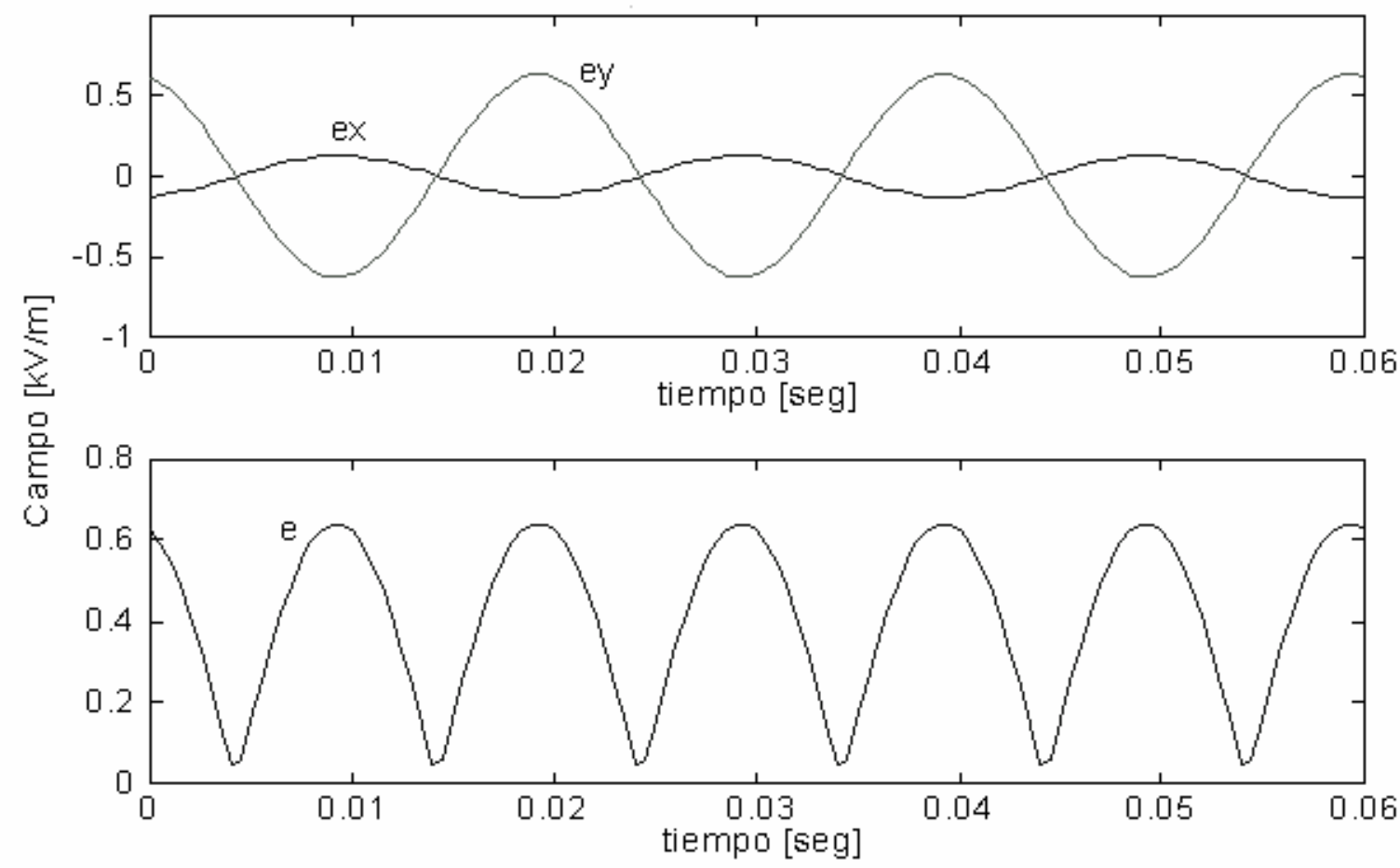

Fig. 3: Variación temporal del valor instantáneo de las componentes $e_{x} y e_{y}$, y del campo resultante $\mathrm{e}$, para un punto en el espacio de coordenadas $(\mathrm{xo}, \mathrm{yo})=(5,1)[\mathrm{m}]$

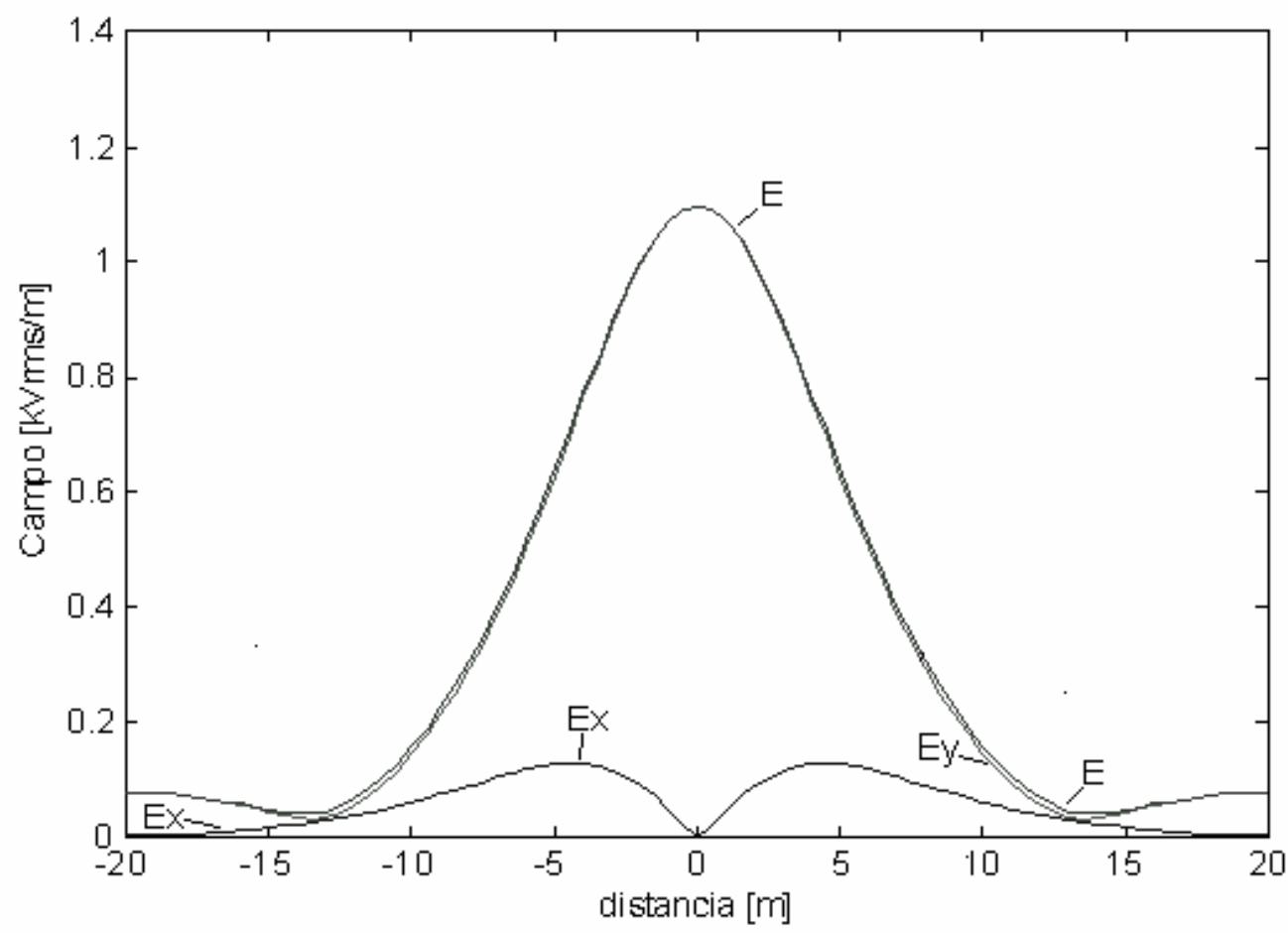

Fig. 4: Variación espacial del vector campo eléctrico resultante E, y sus componentes Ex y Ey.

En particular, se analizó el comportamiento de la línea trifásica del ejemplo mostrado para diferentes configuraciones de sus conductores. Las correspondientes salidas gráficas (no mostradas aquí) resultaron concordantes, en forma, con las provistas por Deno (1976), Vernieri et al. (2002) y Arnera 
et al. (2003). Se observó que la influencia de la componente ex del campo resulta, a la altura considerada (1 [m]), de mucha menor importancia que la componente ey, lo que se evidencia particularmente en los gráficos espaciales donde las curvas de ey y e resultan casi coincidentes. Este es un punto importante a tener en cuenta al momento de realizar mediciones con equipameinto específico, ya que los resultados estarían dando información en magnitud, tanto de la resultante, como de la componente vertical del campo eléctrico.

\section{CONCLUSIONES}

Puede concluirse que la herramienta de software utilizada facilita, mediante las salidas gráficas, la comprensión conceptual del modelo matemático utilizado para el análisis del comportamiento del campo eléctrico producido por líneas de energía.

Además, esta aplicación presenta ventajas para la formación del alumno en este tipo de herramientas computacionales, debido a que le permite explorar diferentes representaciones y estructuras de datos asociados al problema. El ingreso de datos permite generar diferentes conjuntos de valores, y de este modo elegir configuraciones para las que se obtienen resultados aceptables desde el punto de vista reglamentario (Resolución ENRE 1724/98, 1998).

\section{REFERENCIAS}

Abdel-Salam M. y E.Z. Abdel-Aziz; "Corona power loss determination on multi-phase power transmission lines", Electric Power Systems Research: 58(2), 123-132 (2001).

Angeloni, J.C.; "Campos Eléctricos y Magnéticos en Líneas de Transmisión", Seminario Tesis de Grado, Dpto. Ing. Eléctrica, Universidad Nacional del Sur, Bahía Blanca, Argentina (2000).

Arnera, P. y J. Vernieri; "Campos eléctricos y magnéticos de baja frecuencia y su efecto en la salud humana. Resultados de investigaciones y aspectos normativos" (en línea), 29 de Oct. 2007, http://www.iitree-unlp.org.ar/pdf, Presentado en SENEGEL 2001: Gestión Técnico-Comercial de Sistemas Eléctricos Desregulados e Interconexión Energética del Cono Sur, Viña del Mar, Chile, Octubre 9-12 (2001).

Arnera, P. y otros cuatro autores "Evaluación de campos eléctricos y magnéticos en instalaciones de la Administración Nacional Eléctrica-Ande. Asunción. Paraguay" (en línea), 29 de Oct. 2007, http://www.iitree-unlp.org.ar/sector\%204.html, Presentado en el X Encuentro Regional LatinoAmericano de la CIGRE, Puerto Iguazú, Argentina, Mayo 18-22 (2003).

Battisti, E. y otros nueve autores; "Preliminary results on the effects of electromagnetic fields ELF and TAMMEF in the human lymphocytes: evaluation of some biological parameters", Clin Ter.: 158(4), 307-12 (2007)

Correia de Barros, M.T., M.E. Almeida, L. Dubé y B. Stein; "A general-purpose transmission line model and its interface with an electromagnetic transients programme", International Journal of Electrical Power \& Energy Systems: 19(4), 249-254 (1997).

Cvetkovic D., E. Jovanov e I. Cosic: "Alterations in human EEG activity caused by extremely low frequency electromagnetic fields”, Conf Proc IEEE Eng Med Biol Soc., 1:3206-9 (2006).

Dawson, T. y otros cinco autores;, "Modeling of Cardiac Pacemaker Interference by Low Frequency Electric Fields" (en línea), 1 de Nov. 2007, http://ieeexplore.ieee.org/iel5/7218/19434/00897843.pdf, Proceedings of the 22"' Annual EMBS International Conference, Chicago, USA, 23-28 Julio (2000a).

Dawson, T. y otros cinco autores; "Pacemaker interference and low frequency electric induction in humans by external fields and electrodes", IEEE Transactions on Biomedical Engineering: 47(9), $1211-1218(2000 b)$. 
Deno, D. W.; “Transmission Line Fields”, IEEE Transactions on Power Apparatus and Systems: 95(5), 1600-1611 (1976).

Havas M., "Intensity of electric and magnetic fields from power lines within the business district of 60 Ontario communities", The Science of the Total Environment: 298 (1),183-206 (2002).

IRPA; "Protection of workers from power frequency electric and magnetic fields", Occupational Safety and health series, A practical Guide, International Labour Office, Geneva: no. 69, pp. 1- 71 (1994).

IEEE Std. 644; IEEE Standard Procedures for Measurement of Power, 22-23 (1994).

Manco, D. y otros cinco autores; "Electric and magnetic fields generated by ac power lines: an application of advanced modelling tools in order to predict exposure levels", Radiation protection dosimetry: 97(4), 359-362 (2001).

Mirotznik, M. S. y D. Prather; "How to Choose EM Software", IEEE Spectrum: (33), 53-58 (1997).

Parraud, R. "Comparative Electric Field Calculations and Measurements" por aprobación del WG03.22 de la CIGRE (Working Group 03, Subcomité 22) (en línea), 30 de Oct. 2007, http://www.integratedsoft.com/papers/techndocs/tech 2g.pdf, Integrated Engineering Software Simulation Tools for Field Solving and Análisis - Technical Papers, USA (1992).

Que, W. y S. Sebo; "Electric Field Distribution in Air: Examples for Various Energized and Grounded Electrode Configurations" (en línea), 30 de Oct. 2007, http://www.integratedsoft.com/Papers/ research/EFD.pdf, Integrated Engineering Software - Simulation Tools for Field Solving and Análisis Technical Papers, USA (2000).

Resolución ENRE 1724/98. Boletín Oficial N² 29.038, 07-12-98, pp. 25-26, Argentina (1998).

Rossi, A.P. y J. L. Montero; "Análisis del comportamiento temporal en estado estacionario del campo eléctrico en un punto genérico del espacio, en presencia de conductores energizados a frecuencia industrial", Revista Electrotécnica de la AEA (Asociación Electrotécnica Argentina), ISSN 0370-7857 (en línea), 2005 (fecha de consulta Oct. 30, 2007; http://www.aea.org.ar/revista/artec_rev_11.htm).

Saadetdin H. y M. Salih Mami; "Computation of corona effects in transmission lines using state-space techniques", Computers \& Electrical Engineering: 29 (5), 603-611 (2003).

Tiebin Zhao,T. y M. Comber; "Calculation of electric field and potential distribution along nonceramic insulators considering the effects of conductors and transmission towers", IEEE Transactions on Power Delivery: 15(1), 313-318 (2000).

Vernieri, J., P. Arnera y C. Massei; "Exposición a campos eléctricos y magnéticos de frecuencia industrial y sus posibles efectos en la salud humana, reglamentaciones de valores límites de exposición: seguimiento y actualización" (en línea), 29 Oct. 2007, http://www.iitreeunlp.org.ar/sector\%204.html, Presentado en el IX Encuentro Regional Latino-Americano de la CIGRE, Foz do Iguaçu, Brasil, Mayo 27-31 (2001).

Vernieri, J., P. Arnera y M. Barbieri; “Aspectos ambientales de campos eléctricos y magnéticos en líneas de alta tensión" (en línea), 29 Oct. 2007, http://www.iitree-unlp.org.ar/sector\%204.html, Presentado en el CIDEL 2002: CONGRESO INTERNACIONAL DE DISTRIBUCION ELECTRICA, Buenos Aires, Argentina, Diciembre 3-5 (2002). 